\title{
Efficient Screening of Pesticide Diazinon-Binding Aptamers Using The Sol-Gel Coated Nanoporous Membrane-Assisted SELEX Process and Next- Generation Sequencing
}

\section{Min-Cheol Lim}

Korea Food Research Institute

Eun Seob Lim

Korea Food Research Institute

Jeong-A Lim

Korea Food Research Institute

Sung-Wook Choi

Korea Food Research Institute

Hyun-Joo Chang ( $\sim$ hjchang@kfri.re.kr)

Korea Food Research Institute

\section{Research Article}

Keywords: Aptamer, Diazinon, Nanoporous membrane, NGS, SELEX

Posted Date: October 21st, 2021

DOI: https://doi.org/10.21203/rs.3.rs-961731/v1

License: (c) (i) This work is licensed under a Creative Commons Attribution 4.0 International License. Read Full License 


\section{Abstract}

\section{Background}

Aptamer-based methods for detecting pesticides are more efficient than antibody-based methods as aptamers have high thermal stability, low molecular weight, easy modification, and low cost. However, only few studies on SELEX performed in combination with next generation sequencing (NGS) to screen aptamers specific for pesticides have been reported. Therefore, this study aimed to develop the systematic evolution of ligands by exponential enrichment (SELEX) process, combined with NGS, to select aptamers specific to the pesticide, diazinon, which was fixed on a sol-gel-coated nanoporous anodized aluminum oxide membrane.

\section{Methods and results}

The frequency of specific nucleotide sequences obtained after SELEX rounds was analyzed using NGS. Nine sequences with the highest frequency after SELEX round 10 followed by NGS were selected and tested to derive their binding affinity with the target, diazinon, through circular dichroism (CD) spectrophotometry. The CD signal difference of the aptamer candidates ranged from 0.13 to 2.242 mdeg between diazinon-only treated and diazinonaptamer-treated samples at a wavelength near $270 \mathrm{~nm}$. Aptamer D-4, which had the highest binding affinity from CD spectrophotometry analysis, showed no cross-reactivity with non-target pesticides, such as baycarb, bifenthrin, and pyridaben, but interacted with the other pesticides, fipronil and 2-phenylphenol.

\section{Conclusions}

The proposed SELEX process combined with NGS for the discovery of aptamers for new targets can shorten the SELEX cycle by reducing the number of SELEX rounds to 10 or less. Therefore, an efficiently selected aptamer is highly expected to be used for the detection of pesticides in foods in the field.

\section{Introduction}

Pesticides are used to prevent and control insects and weeds to promote crop and other food productivity in recent agriculture [1]. However, due to the overuse of pesticides, the presence of pesticide residues in food, water, and the environment causes serious food contamination, which creates a potential hazard to human health, environmental pollution, and severe breakdown of the ecosystem [2]. Pesticides have adverse effects on human health, such as nausea, dizziness, difficulty breathing, numbness, decreased coordination, slow heartbeat, and headache in the short term and accumulation of pesticides inside the body in the long term $[3,4]$.

The overuse of pesticides has also impacted livestock farms, crops, and other agricultural foods. Pesticidecontaminated egg scares were reported in Europe in 2017. Thereafter, the Ministry of Agriculture, Food and Rural Affairs in Korea revealed that fipronil and bifenthrin were detected in food eggs in eco-friendly laying hen farms. As part of the management of pesticide residues, the Rural Development Administration (RDA) in Korea introduced the positive list system (PLS) for all agricultural products in 2019, and applied $0.01 \mathrm{ppm}$ uniformly to pesticides for which maximal residual levels (MRLs) have not been established. In addition, the Ministry of Food and Drug Safety (MFDS) in Korea regularly monitors pesticide residues in all foods; however, there are several cases of the pesticide residue in food exceed the MRL every year. For example, MFDS monitored 322 cases of high-consumption agricultural products that were distributed and sold in traditional markets and marts nationwide from March 22 to April 6, 2021. Pesticides such as diazinon were found to exceed MRLs in four cases. Diazinon, a target molecule in 
this study, is an organophosphorus pesticide that has been approved for use in both agricultural and livestock products.

Conventionally, pesticides are detected by gas and liquid chromatography, which have high selectivity and sensitivity at low detection limits. However, these methods are laborious, require highly skilled manpower, and utilize costly instruments. Accordingly, an advanced approach to determine pesticides has been reported using sensor-based techniques, which offer several advantages including low-cost, simple, rapid operation, sensitive, and selective on-site detection [5]. Compared with antibody or enzyme-based general biosensors for the detection of pesticide residues as small molecules, aptasensors exhibit better specificity and sensitivity, higher stability, more flexibility, and easier artificial synthesis $[6,7]$.

Aptamers are short single-stranded oligonucleotides (RNA or DNA) that can selectively bind to their targets with high affinity developed through systematic evolution of ligands by exponential enrichment (SELEX) method. Accordingly, aptamers are an ideal alternative for developing biosensors [8, 9]. SELEX experiments are rarely performed with few rounds, possibly because of the non-ideal partition and pool amplification bias [10]. To identify the oligonucleotide sequences selected after the final round of SELEX, cloning and Sanger sequencing are commonly employed. However, Sanger sequencing in the field of aptamer development has been replaced by highthroughput methods, with great progress made in next-generation sequencing (NGS) technologies in the last decade. NGS enables the rapid characterization of millions of aptamer sequences, and sequence data are combined with bioinformatics to select the best aptamer candidates for functional testing [10-12]. Only few reports of studies on SELEX performed in combination with NGS to screen aptamers specific for pesticides have been published.

Selecting aptamers interacting with small molecules as pesticides is accompanied by some specific problems as the possible interactions between the functional groups of the ligand and the aptamer are markedly more limited owing to their small size. One of its functional groups will have to be used for immobilization, thereby further decreasing the number of possible interactions with a future aptamer [13]. We developed a new technique for binding pesticides in a sol-gel coated-anodized aluminum oxide (AAO) membrane to solve the problem of immobilization of the target pesticide in our previous study [14].

In this study, SELEX was performed against the target pesticide, diazinon, using a sol-gel coated AAO membrane. Thereafter, the distribution of aptamers amplified in the SELEX round was investigated by NGS. Among them, aptamers with high frequency were selected and synthesized to measure their binding activity with diazinon using a circular dichroism (CD) spectrum. The proposed approach could save time and labor in the SELEX process, and the selected aptamers could be used for the detection of pesticides in food applications in the future.

\section{Materials And Methods}

\section{Chemicals and kits}

The pesticides diazinon, baycarb, bifenthrin, pyridaben, fipronil, and 2-phenylphenol were purchased from AccuStandard (New Haven, CT, USA). An AAO membrane (Anodisc, 200 nm pore diameter, 25 mm disc diameter, 60 $\mu \mathrm{m}$ thickness) was obtained from Whatman (Maidstone, UK). The three sizes of random DNA templates, 66-mer oligonucleotides [5' TAGGGAAGAGAAGGACATATGAT (N20) TTGACTAGTACATGACCACTTGA 3'], 76-mer oligonucleotides [5' TAGGGAAGAGAAGGACATATGAT (N30) TTGACTAGTACATGACCACTTGA 3'], and 86-mer oligonucleotides [5' TAGGGAAGAGAAGGACATATGAT (N40) TTGACTAGTACATGACCACTTGA 3'], where N indicates 
A, C, G, T wobble site, and forward selection primer [5' TAGGGAAGAGAAGGACATATGAT 3'] and reverse selection primer [5' (Biotin BB ${ }^{\text {TM }}$ ) TCAAGTGGTCATGTACTAGTCAA 3'] were purchased from TriLink biotechnologies (San Diego, CA, USA). Yeast tRNA and streptavidin agarose columns were obtained from Thermo Scientific (Waltham, MA, USA). PCR Premix (Promega, Madison, WI, USA) was used for PCR. The NAPTM 5 column (Sephadex G-25 DNA Grade) was obtained from GE Healthcare (Chicago, IL, USA). The 15\% Mini-PROTEAN TBE-Urea precast gel was purchased from Bio-Rad (Hercules, CA, USA). Dimethylformamide (DMF, 99\%) was purchased from Sigma-Aldrich (St. Louis, MO, USA). SELEX buffer (2×, 100 mM Tri-HCl, pH 7.5, 300 mM NaCl, 50 mM KCl, 0.02\% Tween-20, 10 mM $\mathrm{MgCl}_{2}, 2 \mathrm{mM} \mathrm{CaCl}_{2}$ ) was purchased from Dongin Biotech (Seoul, Korea). Oligonucleotides were synthesized as aptamer candidates from Cosmo Genetech (Seoul, Korea).

\section{Sol-Gel coating on the AAO membrane and the SELEX process}

Sol-gel was coated on the nanoporous AAO membrane to entrap the target pesticide within the sol-gel matrix to prepare the SELEX platform. The overall coating process is described in our previous report [14]. Briefly, the sol-gel composition and preparation method was performed according to the manufacturer's manual with the SolB complete kit (PCL Inc., Seoul, Korea). Diazinon dissolved in 50\% DMF was diluted to 100 ppm, and the prepared sol-gel mixture was mixed at a volume ratio of 1:7. Eighty microliters of diazinon-containing sol-gel mixture was deposited via a single-step spin-coating process at 3,000 rpm for $30 \mathrm{~s}$ onto the AAO membrane fixed to a spin coater (ACE-200, Dong Ah Tech, Seoul, Korea). After gelation of the sol-gel-coated AAO membrane overnight at high humidity $(90 \% \mathrm{RH})$ and room temperature, it was cut with a $6 \mathrm{~mm}$ punch (Acu-punch; Acuderm, Fort Lauderdale, FL, USA) for subsequent use.

The sol-gel-coated AAO membrane immobilized with diazinon was soaked in $200 \mu \mathrm{l} \mathrm{of} 20 \mu \mathrm{g} / \mathrm{ml}$ yeast tRNA for $2 \mathrm{~h}$ at room temperature, and then washed with $1 \times$ PBS $(0.2 \%$ Tween-20). The three sizes of DNA templates were mixed at a concentration of $1 \mu \mathrm{M}$ to create a random aptamer library. Thereafter, the tRNA-treated sol-gel-coated AAO membrane was soaked in $200 \mu \mathrm{l}$ of $1 \mu \mathrm{M}$ random aptamer library for $1 \mathrm{~h}$ at room temperature. The membrane was washed with PBS and kept in $50 \mu$ of distilled water for 5 min at $95^{\circ} \mathrm{C}$. The eluted DNA was amplified by PCR in a premixed solution with selection primers. PCR was carried out for 25 cycles, consisting of denaturation at 94 ${ }^{\circ} \mathrm{C}$ for $30 \mathrm{~s}$, annealing at $54{ }^{\circ} \mathrm{C}$ for $30 \mathrm{~s}$, and extension at $72{ }^{\circ} \mathrm{C}$ for $30 \mathrm{~s}$. PCR products were identified using $5 \%$ agarose gel electrophoresis.

For ssDNA separation, the PCR product was loaded onto a streptavidin column, and subsequently washed with $1 \times$ PBS. After the elution step with $2 \mathrm{ml}$ of $0.2 \mathrm{M} \mathrm{NaOH}$, the eluate was neutralized with $1 \mathrm{~N} \mathrm{HCl}$ and loaded onto a NAP-5 column for buffer exchange. SELEX buffer was used for elution. The eluate was identified by $15 \%$ MiniPROTEAN TBE-Urea gel electrophoresis and used for subsequent SELEX rounds.

\section{NGS and data analysis}

Paired-end sequencing was performed on a MiSeq using the Illumina platform at Macrogen (Seoul, Korea), generating $301 \mathrm{bp}$ reads per end, according to the 16S Metagenomic Sequencing library preparation Part \#15044223 Rev. B protocol and Herculase II Fusion DNA Polymerase Nextera XT Index Kit V2. For library construction, six eluates (buffer-exchanged SsDNA), generated by the number of SELEX rounds $(0,2,4,6,8,10)$ were used for DNA quality control (QC). After QC of DNA, the sequencing library was prepared by random fragmentation of the DNA sample, followed by 5 ' and 3 ' adaptor ligation. Adapter-ligated fragments were then PCRamplified and gel-purified. For cluster generation, the library was loaded into a flow cell where fragments were 
captured on a lawn of surface-bound oligos complementary to the library adapters. Each fragment was then amplified into distinct clonal clusters through bridge amplification. The obtained sequencing data were converted into raw data for analysis. The Illumina sequencer generates raw images utilizing sequencing control software for system control and base calling through an integrated primary analysis software called Real Time Analysis (RTA). The BCL (base calls) binary was converted into FASTQ utilizing the illumine package, bcl2fastq. Adapters were not trimmed from the reads. K-mer was analyzed from the raw data.

\section{Pesticide-aptamer binding assay}

The CD spectrum was generated to characterize the interaction between the pesticide and aptamer candidate using a CD spectrometer (J-1500, Jasco, Japan). The wavelength range for analysis was 400-180 nm, and the scanning speed was $100 \mathrm{~nm} / \mathrm{min}$. Diazinon $(66 \mu \mathrm{M})$ and aptamer $(3 \mu \mathrm{M})$ were solubilized in $1 \times$ SELEX buffer. The total volume used in each experiment was $300 \mu \mathrm{l}$ and the methanol concentration was adjusted to $20 \%$. The binding affinity between diazinon and the aptamer candidate was considered as the CD signal difference (mdeg), which represents the values calculated by subtracting a CD signal after binding of diazinon and the aptamer candidate with that of the aptamer candidate without pesticide at a wavelength near $270 \mathrm{~nm}$.

\section{Results}

\section{Aptamer selection through the SELEX process using sol-gel coated nanoporous membrane}

To screen target-specific aptamers from a random aptamer library, 10 selection rounds were performed. The library was reacted with target pesticide immobilized sol-gel AAO membrane, and then the eluted DNA was amplified by PCR and confirmed by 5\% DNA gel electrophoresis (data not shown). To track the selection process, we monitored the PCR products of every selection round to analyze the pools for diversity using TBE-urea gel electrophoresis. TBE-urea gel maintains denaturing conditions for analysis of single-stranded DNA and RNA, and nucleic acids between 60 and 200 bases are resolved as sharp and distinct bands. The biotin-conjugated single-stranded DNA was separated by passing through a streptavidin column and buffer-exchanged, as shown in Fig. 1. Three sizes of amplified DNA bands appeared on the 15\% TBE-urea gel, which were smaller than the actual sizes, 66, 76, and 86 bp of DNA in the random library, respectively. This size gap is due to the difference in running patterns between the double-stranded DNA ladder and the single-stranded target DNA sample in gel electrophoresis. The band intensity of the 66 bp oligonucleotides gradually weakened from round 3 , and the strength of all three bands decreased as the rounds were repeated. From round 8 , the new PCR product with a markedly larger size than the target DNAs began to be amplified, and toward rounds 9 and 10, the band intensity of the new PCR product became stronger. The abnormally large bands shown in rounds 9 and 10 are considered PCR artifacts due to aberrant replication, which can also be confirmed from the K-mer distribution analysis of NGS data, as shown in Fig. 2.

\section{NGS analysis}

Purified DNA samples from even-numbered SELEX rounds were subjected to QC for NGS analysis. The DNA concentration in each sample that passed QC ranged from 0.052 to $0.296 \mu \mathrm{g}$. The raw data statistics after NGS analysis are presented in Table 1. Total reads gradually increased from 3,330,240 (round 0) to 4,056,658 (round 6) and then decreased to $2,878,964$ in round 10 . As the SELEX round progressed, the GC content and phred quality score, Q20 and Q30, gradually increased. The phred quality score numerically expresses the accuracy of each nucleotide, and a high Q number indicates higher accuracy. If phred assigns a quality score of 30 to a base, the 
chance of having a base call error is 1 in 1,000, similar to $99.9 \%$ of base call accuracy. Similarly, Q20 represents $99 \%$ of the base call accuracy.

The distributions of K-mer in samples even after the SELEX round are shown in Fig. 2. In round 0, 61-70 mer, 7180 mer, and 81-90 mer were distributed in similar proportions at 32.3\%, 30.4\%, and 36.9\%, respectively; however, in round 2, 81-90 mer increased sharply to 50.4\%, and 61-70 mer decreased rapidly to $13.2 \%$. The $81-90$ mer showed a peak at $66.7 \%$ of the highest value in round 6 and then gradually decreased. The $71-80$ mer was distributed at a similar rate in all rounds. The ratio of 121-480 mer oligonucleotides with non-target size started to increase to $14.9 \%$ in round 8 and reached $30 \%$ in round 10 . After NGS raw data analysis, nine oligonucleotides with the highest frequency in round 10 were selected as aptamer candidates, and the percentage of each candidate in round 10 ranged from $0.302-3.171 \%$ (Table 2 ). All nine oligonucleotides were hardly amplified until round 6; however, their counts started to increase in round 8 and sharply increased in round 10 (Fig. 3).

\section{Affinity and specificity analysis of aptamer against target pesticide}

The binding properties between the aptamer candidates and the target diazinon were measured by CD spectroscopy. The CD spectrum of the aptamer itself had a negative peak in the wavelength range of $240-250 \mathrm{~nm}$ and a positive peak at 260-280 nm. When aptamer candidates were treated with diazinon, the negative peak decreased significantly and the positive peak increased significantly, as shown for the D-4 aptamer (Fig. 4). Among the nine aptamer candidates, most represented a CD signal difference of $0.13-2.242$ between the aptamer-only sample and pesticide-treated aptamer sample at a wavelength of approximately $270 \mathrm{~nm}$, except for D-2 (Table 2).

The cross-reactivity of the diazinon-binding aptamer against non-target pesticides was also investigated by CD spectrum analysis, as described above. When the binding affinities between the D-4 aptamer and diazinon were compared with those of non-target pesticides, fipronil and 2-phenylphenol showed binding affinities of $89.2 \%$ and $63.8 \%$, respectively, whereas no cross-reactivity was observed for baycarb, bifenthrin, and pyridaben (Fig. 5).

\section{Discussion}

The SELEX process is accompanied by extensive enzymatic amplification. In the course of a multi-cyclic SELEX experiment, the evolution pressure shifts from the side of high-affinity sequences to sequences that are better amplifying $[11,15,16]$. This amplification bias favors shorter and structurally less stable sequences [17]. In the case of diazinon, this negative effect did not appear until SELEX round 7. Thus, the separation of target-bound sequences from those with no affinity for the target is a critical step in the SELEX process [18]. It is necessary to screen selective aptamers for small target molecules, such as pesticides, and a technical challenge is target immobilization while retaining their native molecular structure. To solve the problem of limited possible interactions of the functional groups between the ligand and the aptamer owing to their small size, we introduced a sol-gel-coated AAO membrane. The wet chemical sol-gel process can trap a pesticide in its nano-sized cavity. Further, the nanoporous AAO membrane increased the binding surface to enhance the efficiency of aptamer screening, and effectively retrieved bound aptamers from the sol-gel matrix by simple thermal elution [14].

We performed 10 rounds of the SELEX process, based on the report that at least 5-15 cycles should be conducted to select the target molecule and minimize PCR errors [10]. However, as the SELEX round progressed toward the second half in this study, the ratio of oligonucleotides larger than the target size increased (Fig. 1 and 2). Through sequence review after NGS analysis, these newly emerging oligonucleotides were mostly found to have repeat 
sequences, representing an altered replication. Accordingly, an increase in the number of SELEX cycles does not necessarily lead to high efficiency.

In our preliminary study, we obtained only 10 clones through multiple cloning attempts and Sanger sequencing after SELEX round 4 (data not shown). If the binding activity with the target is assumed to be measured, but none had any activity, SELEX cycles followed by cloning and Sanger sequencing would have to be repeated to identify new candidates. The cloning step is time-consuming because of its complicated process and low probability. Therefore, direct analysis of the oligonucleotide sequence by NGS and selection of aptamer candidates depending on the proportion effectively reduce the SELEX process. The Illumina SBS technology used in this study utilizes a proprietary reversible terminator-based method that detects single bases as they are incorporated into DNA template strands. As all four reversible, terminator-bound dNTPs are present during each sequencing cycle, natural competition minimizes incorporation bias and markedly reduces raw error rates compared to other technologies. This highly accurate base-by-base sequencing virtually eliminates sequence-context-specific errors, even within repetitive sequence regions and homopolymers. Thus, several candidates with sequence information were sufficiently secured through NGS after SELEX.

From NGS data analysis, the GC \% of the obtained sample gradually increased depending on the repeated SELEX process, as shown in Table 1. Thus, the GC \% of the eight aptamer candidates, except D-5, was higher than that of the average GC \% of sample R10 in Table 1. The elevated GC content improves the stability of the aptamer, leading to a stem-rich structure [19]. From the CD spectrum analysis, the D-4 aptamer-treated sample showed the largest CD signal difference of 2.242 and was selected for specificity analysis (Table 2 and Fig. 4).

The target-specific binding ability of aptamers is an important requirement for the development of single-target detection. However, the simultaneous detection of multiple components is important for pesticides in food and environmental samples [20]. Therefore, the cross-reactivity of the aptamer is advantageous for the detection of target families by mixing a set of appropriate aptamers [21]. CD spectrum analysis is a useful tool for monitoring DNA conformational changes resulting from the interaction between DNA and small molecules [22]. D-1 and D-2 aptamer candidates had high frequency in the NGS data of SELEX round 10, but did not have a low binding affinity with the target, diazinon. Such finding suggests that the concentration of the target DNA amplified by repeating the SELEX cycle is not necessarily proportional to the target-selective binding activity. Therefore, a binding test should be performed to verify the aptamer candidate with high frequency. Thus, CD analysis is useful for identifying the cross-reactivity of the aptamer candidate with other pesticides and to construct target lists and secure multiple detection strategies.

Determination of the optimal rounds of SELEX may vary depending on the target molecule. In our study, the D-4 aptamer, which had the highest affinity as measured by CD, showed a low frequency of $0.02 \%$ in round 8; however, its frequency increased rapidly to $0.499 \%$ in round 10 (Fig. 3). Schütze et al performed 10 rounds of aptamer selection from a DNA library using streptavidin-covered magnetic beads as a model target, and carried out NGS with the Illumina platform [23]. In that study, among the aptamers selected in round 10, the aptamer with the highest affinity as measured by surface plasmon resonance (SPR) was identified in the very early round (1-2) and exhibited the highest frequency in round 7. Based on these results, aptamer selection is possible through SELEX at a round earlier than round 10 through NGS, depending on the target. Therefore, a follow-up study is required to verify the proposed approach. 
Sensor development as a pesticide detection method is progressing steadily despite the opinion that it is not suitable for pesticides as hundreds of pesticides exist. As the D-4 aptamer selected for diazinon, an organophosphorus pesticide, had cross-reactivity with fipronil and 2-phenylphenol, which has antifungal activity, this could be advantageous for the simultaneous detection of various types of pesticides. Thus, 2-phenylphenol is not designated as a fungicide in Korea; however, it has been approved in Europe and is applied to citrus fruits. Accordingly, it may cause problems when imported as a contaminant. Therefore, binding spectrum studies against various pesticides should be conducted in the near future for the simultaneous detection of multiple pesticides.

\section{Declarations}

\section{Acknowledgments}

This research was supported by the Main Research Program (E0210701-01) of the Korea Food Research Institute (KFRI) funded by the Ministry of Science and ICT.

\section{Author contributions}

MC, SW, and HJ conceived and designed the experiments. MC, ES, and JA performed the experiments. JA and SW analyzed data and $\mathrm{MC}$ and $\mathrm{HJ}$ wrote the manuscript. All authors read and approved the final manuscript.

\section{Corresponding authors}

Correspondence to Sung-Wook Choi or Hyun-Joo Chang.

\section{Funding}

This study was funded by Ministry of Science and ICT (Grant No. E0210701-01).

\section{Ethics declarations}

\section{Conflict of interests}

The authors declare that they have no conflict of interest associated with the report of the present study.

\section{References}

1. Songa EA, Okonkwo JO (2016) Recent approaches to improving selectivity and sensitivity of enzyme-based biosensor for organophosphorus pesticides: A review. Talanta 155:289-304

2. Campanella L, Colapicchioni C, Favero G, Sammartino MP, Tomassetti M (1996) Organophosphorus pesticide (Paraoxon) analysis using solid state sensor. Sens Actuators B Chem 33:25-33

3. Valcke M, Bourgault M-H, Rochette L, Normandin L, Samuel O, Belleville D, Blanchet C, Phaneuf D (2017) Human health risk assessment on the consumption of fruits and vegetables containing residual pesticides: $A$ cancer and non-cancer risk/benefit perspective. Environ Int 108:63-74

4. Hashwan SSB, Khir MHBM, Al-Douri Y, Ahmed AY (2020) Recent Progress in the development of biosensors for chemicals and pesticides detection. IEEE Access 8:82514-82527

5. Narenderan ST, Meyyanathan SN, Babu B (2020) Review of pesticide residue analysis in fruits and vegetables. Pre-treatment, extraction and detection techniques. Food Res Intern 133:109141:1-10 
6. Bagheri E, Abnous K, Alibolandi M, Ramezani (2018) Triple-helix molecular switch-based aptasensors and DNA sensors. Biosens Bioelectron 111:1-9

7. Kim YS, Raston NHA, Gu MB (2016) Aptamer-based nanobiosensors. Biosens Bioelectron 76:2-19

8. Liu M, Khan A, Wang Z, Liu Y, Yang G, Deng Y, He N (2019) Aptasensors for pesticide detection. Biosens Bioelectron 130:174-184

9. Darmostuk M, Rimpelova S, Gbelcova H, Rumi T (2015) Current approaches in SELEX: An update to aptamer selection technology. Biotechnol adv 33:1141-1161

10. Komarova N, Kuznetsov A (2019) Inside the black box: What makes SELEX better? Molecules 24:3598:1-23

11. Blind M, Blank M (2015) Aptamer selection technology and recent advances. Mol Ther-Nucleic acids 4:e223

12. Komarova N, Barkova D, Kuznetsov A (2020) Implementation of high-throughput sequencing (HTS) in aptamer selection technology. Int J Mol Sci 21:8774-8795

13. Pfeiffer F, Mayer G (2016) Selection and biosensor application of aptamers for small molecules. Front Chem 4:25

14. Lim ES, Lim M-C, Park K, Lee G, Lim J-A, Woo M-A, Lee N, Choi S-W, Chang H-J (2020) Selective binding and elution of aptamers for pesticide based on sol-gel coated nanoporous anodized aluminum oxide membrane. Nanomaterials 10:1533-1543

15. Cho M, Xiao Y, Nie J, Stewart R, Csordas AT, Oh SS, Thomson JA, Soh HT (2010) Quantitative selection of DNA aptamers through microfluidic selection and high-throughput sequencing. Proc Natl Acad Sci ISA 107:1537315378

16. Kupakuwana GV, Crill JE 2nd, McPike MP, Borer PN (2011) Acyclic identification of aptamers for human alphathrombin using over-represented libraries and deep sequencing. PloS One 6: e19395:1-11

17. Zimmermann B, Bilusic I, Lorenz C, Scnroeder R (2011) Genomic SELEX: A discovery tool for genomic aptamers. Methods 52:125-132

18. McKeaque M, DeRosa MC (2012) Challenges and opportunities for small molecule aptamer development. J Nucleic acids 2012:748913:1-21

19. Adachi T, Nakamura Y (2019) Aptamers: A review of their chemical properties and modifications for therapeutic application. Molecules 24(23):4229

20. Song N-E, Lee JY, Mansur AR, Jang HW, Lim M-C, Lee Y, Yoo M, Nam TG (2019) Determination of 60 pesticides in hen eggs using the QuEChERS procedure followed by LC-MS/MS and GC-MS/MS. Food Chem 298:125050

21. Liu Y, Yu H, Alkhamis O, Moliver J, Xiao Y (2020) Tuning biosensor cross-reactivity using aptamer mixtures. Anal Chem 92(7):5041-5047

22. Zhou X, Wang L, Shen G, Zhang D, Xie J, Mamut A, Huang W, Zhou S (2018) Colorimetric determination of ofloxacin using unmodified aptamers and the aggregation of gold nanoparticles. Microchim Acta 185:355363

23. Schütze T, Wilhelm B, Greiner N, Braun H, Peter F, Mörl M, Erdmann VA, Lehrach H, Konthur Z, Menger M, Arndt PF, Glökler J (2011) Probing the SELEX process with next-generation sequencing. PLoS ONE 6:e29604

\section{Tables}


Table 1

Raw data statistics from NGS for the PCR products after SELEX targeting diazinon

\begin{tabular}{|lllllll|}
\hline Sample & Total read bases (bp) & Total reads & GC(\%) & AT(\%) & Q20(\%) & Q30(\%) \\
\hline R0 & $1,002,402,240$ & $3,330,240$ & 35.65 & 64.35 & 59.40 & 49.10 \\
\hline R2 & $1,104,474,350$ & $3,669,350$ & 36.95 & 63.05 & 59.88 & 49.95 \\
\hline R4 & $1,125,146,428$ & $3,738,028$ & 36.57 & 63.43 & 60.89 & 51.13 \\
\hline R6 & $1,221,054,058$ & $4,056,658$ & 37.55 & 62.45 & 60.71 & 50.98 \\
\hline R8 & $1,146,303,718$ & $3,808,318$ & 39.64 & 60.36 & 61.27 & 50.88 \\
\hline R10 & $866,568,164$ & $2,878,964$ & 39.29 & 60.71 & 64.08 & 52.84 \\
\hline
\end{tabular}

Total read bases : Total number of bases sequenced

Total reads : Total number of reads. For Illumina paired-end sequencing, this value refers to the sum of reads 1 and 2.

$\mathrm{GC}(\%)$ : GC content

AT(\%) : AT content

Q20(\%) : Ratio of bases that have a phred quality score greater than 20

Q30(\%) : Ratio of bases that have a phred quality score greater than 30 
Table 2

List of diazinon-binding aptamer candidates identified by Illumina sequencing after 10 selection rounds

\begin{tabular}{|c|c|c|c|c|}
\hline Aptamer & Sequence & $\begin{array}{l}\text { Length } \\
\text { (mer) }\end{array}$ & $\begin{array}{l}\text { Percentage } \\
\text { in the } 10 \text { th } \\
\text { selection } \\
\text { round (\%) }\end{array}$ & $\begin{array}{l}\text { CD signal } \\
\text { difference } \\
\text { (mdeg)* }\end{array}$ \\
\hline \multirow[t]{2}{*}{ D-1 } & 5'-TAGGGAAGAGAAGGACATATGATGGCAAGCACCGGAGGT & 76 & 3.171 & 0.13 \\
\hline & TGGTGGTGGGTTGTTTGACTAGTACATGACCACTTGA-3’ & & & \\
\hline \multirow[t]{2}{*}{ D-2 } & 5'-TAGGGAAGAGAAGGACATATGATGCGGTCTCAGAAATGC & 76 & 0.916 & 0 \\
\hline & GATTGATGCGGCGGTTGACTAGTACATGACCACTTGA-3' & & & \\
\hline \multirow[t]{2}{*}{ D-3 } & 5'TAGGGAAGAGAAGGACATATGATGGATAGTCCATGGACGC & 76 & 0.549 & 1.217 \\
\hline & AAGCGTGTGTTGGTTGACTAGTACATGACCACTTGA-3’ & & & \\
\hline \multirow[t]{2}{*}{ D-4 } & 5'-TAGGGAAGAGAAGGACATATGATAGATCATATCGATAGCAT & 76 & 0.499 & 2.242 \\
\hline & CTTCCTCACAGGTTGACTAGTACATGACCACTTGA-3' & & & \\
\hline \multirow[t]{2}{*}{ D-5 } & 5'-TAGGGAAGAGAAGGACATATGATCTGTTGTTGTTGCTATT & 64 & 0.471 & 0.51 \\
\hline & CGATTGACTAGTACATGACCACTT-3’ & & & \\
\hline \multirow[t]{2}{*}{ D-6 } & 5'-TAGGGAAGAGAAGGACATATGATTCTGGGTCAAAGGGGGA & 76 & 0.403 & 1.097 \\
\hline & TAGTTAGCGTTGGTTGACTAGTACATGACCACTTGA-3' & & & \\
\hline \multirow[t]{2}{*}{ D-7 } & 5'-TAGGGAAGAGAAGGACATATGATGTTTGGGTTAATGCACA & 66 & 0.368 & 1.771 \\
\hline & TTGTTGACTAGTACATGACCACTTGA-3' & & & \\
\hline \multirow[t]{3}{*}{ D-8 } & 5'-TAGGGAAGAGAAGGACATATGATGGTAAACTTGTGGCGTA & 86 & 0.361 & 1.696 \\
\hline & CATCTTCATAACATCAGGTCGCATTGACTAGTACATGACCACT & & & \\
\hline & TGA-3' & & & \\
\hline \multirow[t]{2}{*}{ D-9 } & 5'-TAGGGAAGAGAAGGACATATGATGGCAGATTATTCATTGG & 76 & 0.302 & 2.154 \\
\hline & AGCTAAACGTGCATTGACTAGTACATGACCACTTGA-3' & & & \\
\hline
\end{tabular}

\section{Figures}




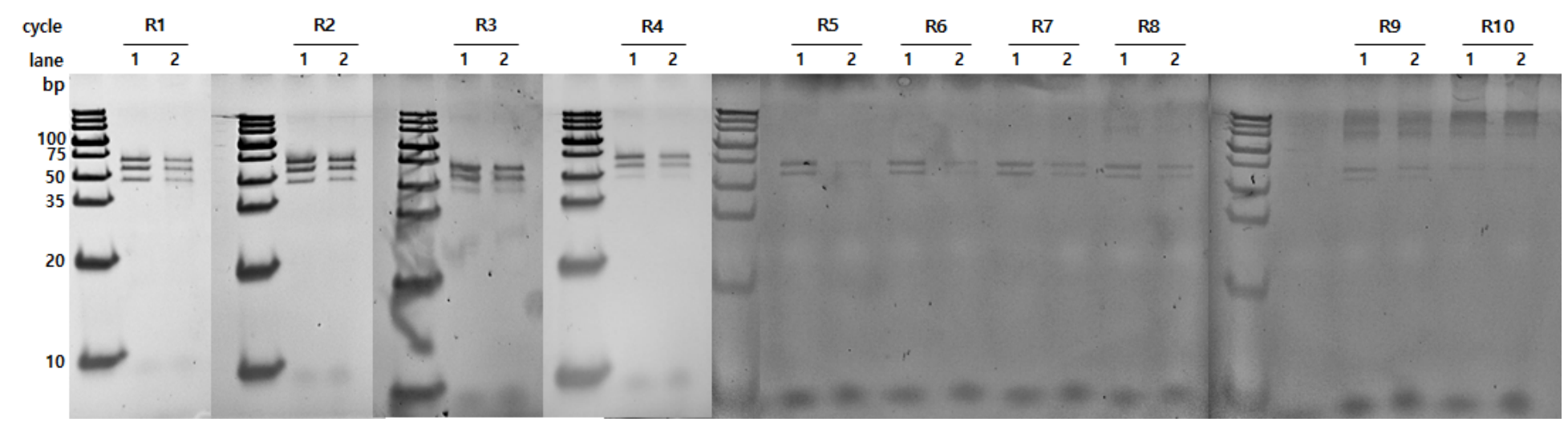

Figure 1

DNA gel electrophoresis of ssDNA aptamer targeting diazinon purified using avidin column in the SELEX process Lane 1: single-strand DNA Lane 2: buffer-exchanged single-strand DNA

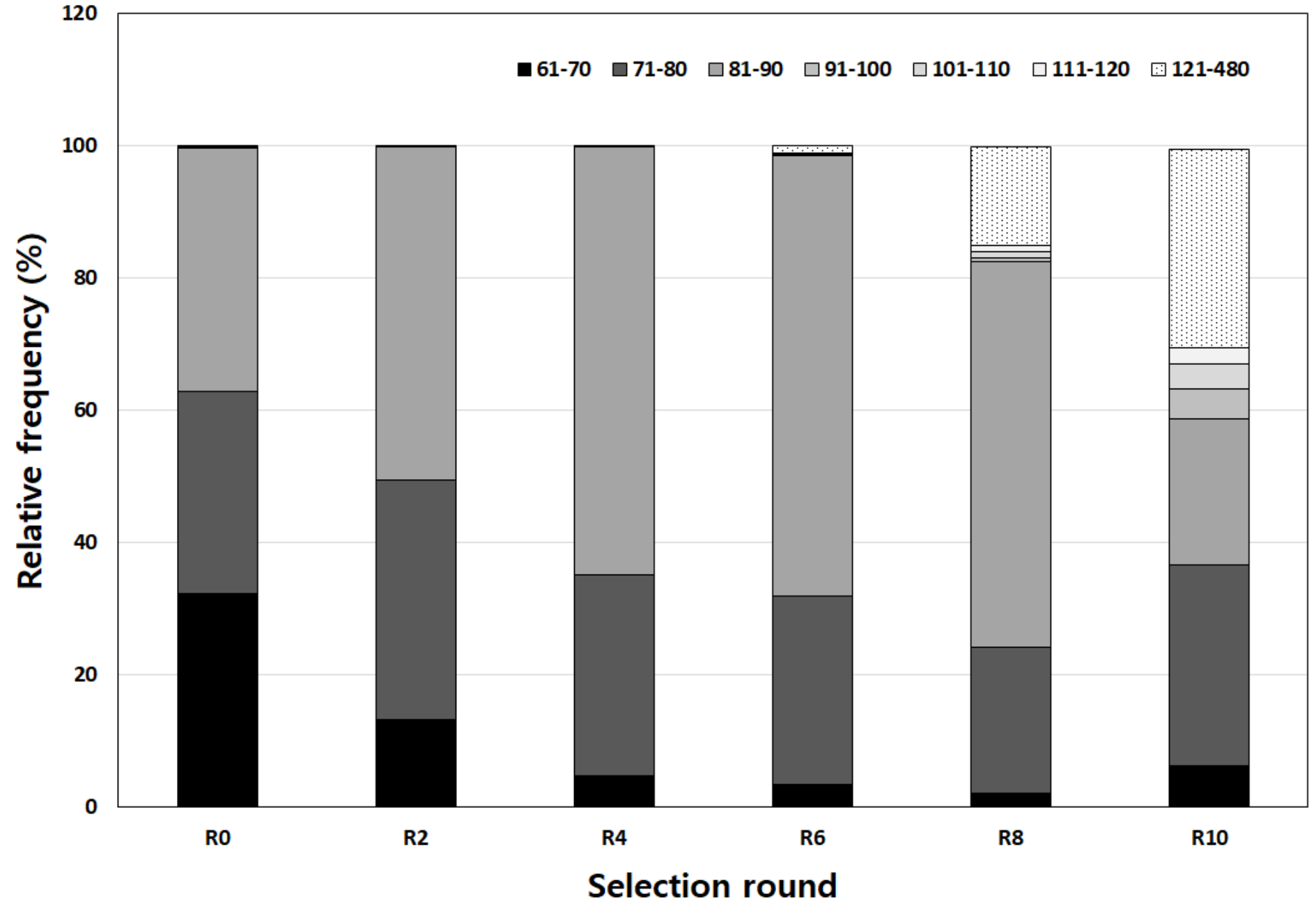

Figure 2

K-mer distribution of amplified diazinon aptamer from next generation sequencing data by selection rounds 


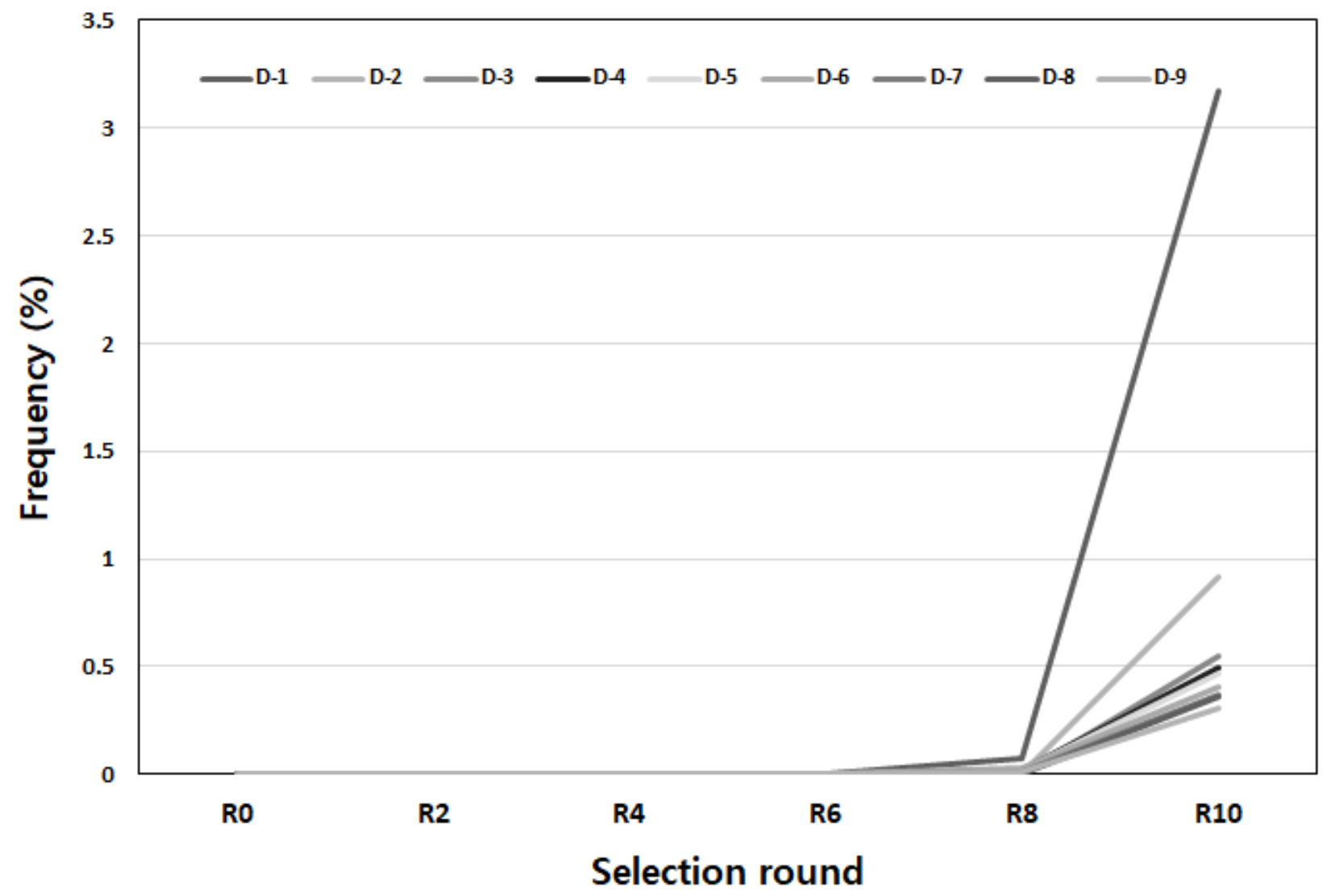

Figure 3

Frequency of nine oligonucleotides by the number of SELEX round after next generation sequencing analysis 


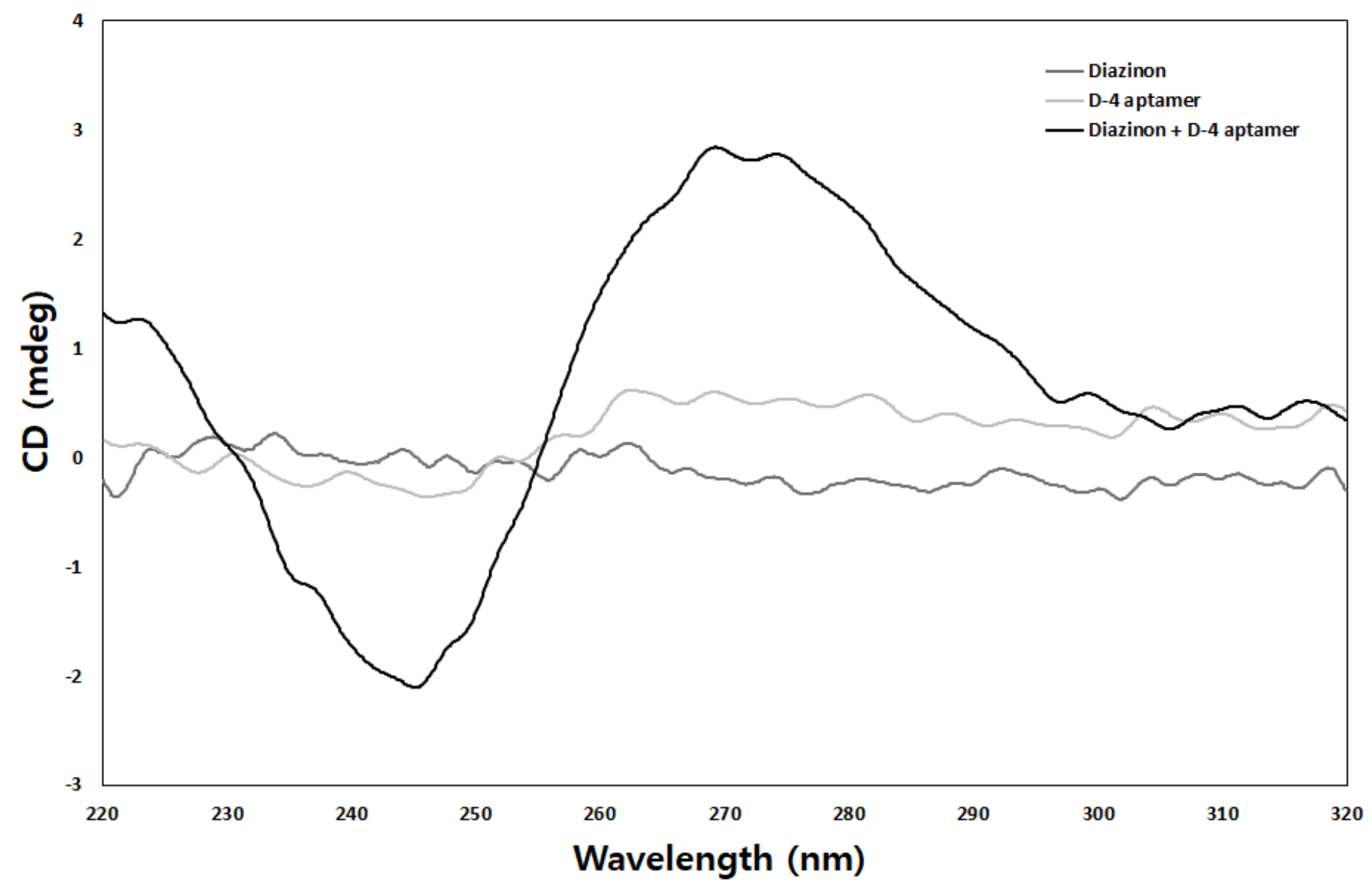

Figure 4

Circular dichroism spectra of the D-4 aptamer treated with diazinon 


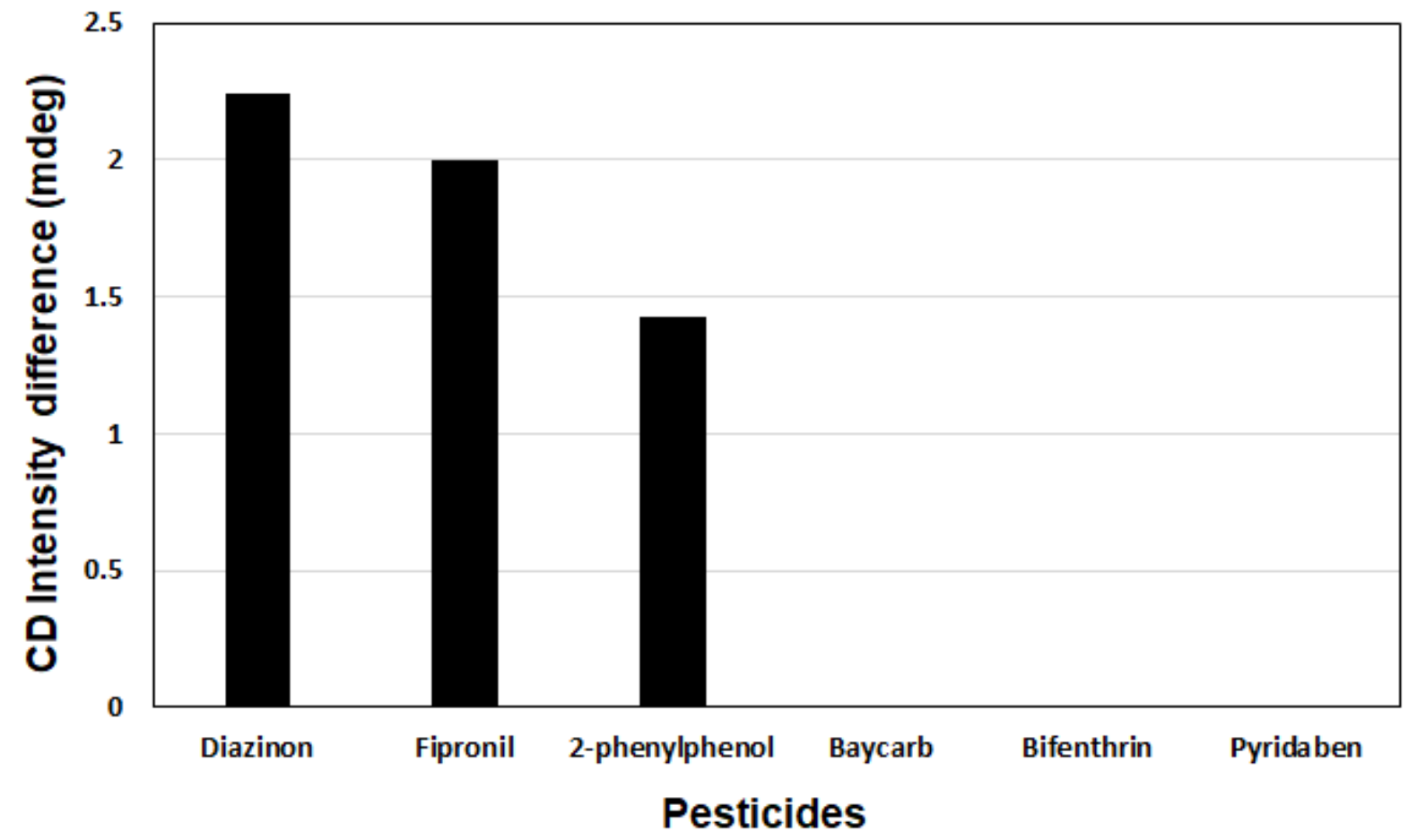

Figure 5

Cross-reactivity of diazinon-binding aptamer against non-target pesticides 